\title{
RAIRO
}

ANALYSE NUMÉRIQUE

\author{
R. S. FALK \\ J. E. OSBORN
}

\section{Error estimates for mixed methods}

RAIRO - Analyse numérique, tome 14, no 3 (1980), p. 249-277.

<http://www.numdam.org/item?id=M2AN_1980_14_3_249_0>

(C) AFCET, 1980, tous droits réservés.

L'accès aux archives de la revue «RAIRO - Analyse numérique » implique l'accord avec les conditions générales d'utilisation (http://www.numdam.org/ legal.php). Toute utilisation commerciale ou impression systématique est constitutive d'une infraction pénale. Toute copie ou impression de ce fichier doit contenir la présente mention de copyright.

\section{Numdam}

Article numérisé dans le cadre du programme

Numérisation de documents anciens mathématiques

http://www.numdam.org/ 


\title{
ERROR ESTIMATES FOR MIXED METHODS ( $\left.{ }^{*}\right)$
}

\author{
by R. S. FALK $\left({ }^{1}\right)$ and J. E. OsborN $\left({ }^{2}\right)$ \\ Communicated by P. A. RaviarT
}

Abstract. - This paper presents abstract error estimates for mixed methods for the approximate solution of elliptic boundary value problems. These estimates are then applied to obtain quasi-optimal error estimates in the usual Sobolev norms for four examples: three mixed methods for the biharmonic problem and a mixed method for second order elliptic problems.

Résumé. - Dans cet article, on présente des estimations d'erreur abstraites pour des méthodes mixtes appliquées à la résolution approchée de problèmes aux limites elliptiques. On applique ensuite ces estimations afin d'obtenir des estimations d'erreur quasi-optimales, dans les normes de Sobolev habituelles, dans quatre exemples: Trois méthodes mixtes pour le problème biharmoniques, et une méthode mixte pour les problèmes elliptiques du second ordre.

\section{INTRODUCTION}

In [5] Brezzi studied Ritz-Galerkin approximation of saddle-point problems arising in connection with Lagrange multipliers. These problems have the form:

Given $f \in V^{\prime}$ and $g \in W^{\prime}$, find $(u, \psi) \in V \times W$ satisfying

$$
\left.\begin{array}{c}
a(u, v)+b(v, \psi)=(f, v), \quad \forall v \in V, \\
b(u, \varphi)=(g, \varphi), \quad \forall \varphi \in W,
\end{array}\right\}
$$

where $V$ and $W$ are real Hilbert spaces, and $a(.,$.$) and b(.,$.$) are bounded$ bilinear forms on $V \times V$ and $V \times W$, respectively.

Given finite dimensional spaces $V_{h} \subset V$ and $W_{h} \subset W, 0<h<1$, the RitzGalerkin approximation $\left(u_{h}, \psi_{h}\right)$ to $(u, \psi)$ is the solution of the following problem:

Find $\left(u_{h}, \psi_{!}\right) \in V_{l .} \times W_{h}$ satisfying

$$
\left.\begin{array}{c}
a\left(u_{h}, v\right)+b\left(v, \psi_{h}\right)=(f, v), \quad \forall v \in V_{h}, \\
b\left(u_{\|}, \varphi\right)=(g, \varphi), \quad \forall \varphi \in W_{h} .
\end{array}\right\}
$$

(*) Received May 1979.

The work of the first author was partially supported by NSF grant MCS78-02737 and by the United States Army under Contract No. DAAG29-75-C-0024, and the second author by NSF Grant MCS78-02851 and by the United States Army under Contract No. DAAG29-75-C-0024.

( $\left.{ }^{1}\right)$ Department of Mathematics, Rutgers University, New Brunswick, New Jersey 08903, U.S.A.

$\left({ }^{2}\right)$ Department of Mathematics, University of Maryland, College Park, Maryland 20742, U.S.A.

R.A.I.R.O. Analyse numérique/Numerical Analysis, 0399-0516/1980/249/\$ 5.00 AFCET-Bordas-Dunod 
The major assumptions in Brezzi's results are

$$
\sup _{v \in Z_{h}} \frac{|a(u, v)|}{\|v\|_{V}} \geqq \gamma_{0}\|u\|_{V}, \quad \forall u \in Z_{h} \quad \text { and } \quad \forall h,
$$

where $\gamma_{0}>0$ is a constant independent of $h$ and

$$
Z_{h} \equiv\left\{v \in V_{h}: b(v, \varphi)=0, \forall \varphi \in W_{h}\right\},
$$

and

$$
\sup _{v \in V_{h}} \frac{|b(v, \varphi)|}{\|v\|_{V}} \geqq k_{0}\|\varphi\|_{W}, \quad \forall \varphi \in W_{h} \text { and } \forall h,
$$

where $k_{0}>0$ is independent of $h$.

Using (1.3) and (1.4) Brezzi proves the following error estimate for the approximation method determined by (1.2):

$\left\|u-u_{h}\right\|_{V}+\left\|\psi-\psi_{I}\right\|_{W} \leqq C\left(\inf _{\chi \in V_{h}}\|u-\chi\|_{V} \quad+\inf _{\eta \in W_{h}}\|\psi-\eta\|_{W}\right), \quad \forall h$.

In $[1,2]$ Babuška studied Ritz-Galerkin approximation of general variationally posed problems. The main result of $[1,2]$, applied to $(1.1)$ and (1.2), is that (1.5) holds provided

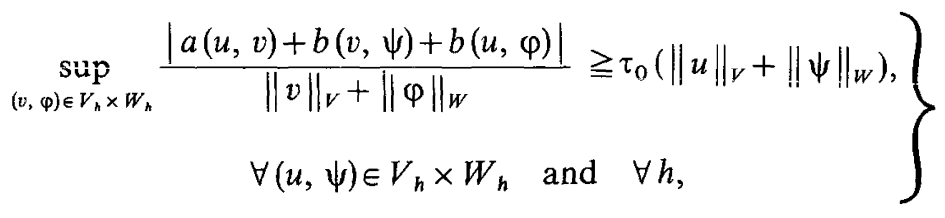

where $\tau_{0}>0$ is independent of $h$.

It is clear from [1, 2, 5] that (1.3) and (1.4) hold if and only if (1.6) holds. (1.3)(1.4) or, equivalently, (1.6) is referred to as the stability condition for this approximation method.

The results of $[1,2,5]$ can be viewed as a strategy for analyzing these approximation methods: the approximation method is characterized by certain bilinear forms, norms (spaces), and families of finite dimensional approximating spaces, and if the method can be shown to be stable with respect to the chosen norms, then the error estimates in these norms follow in a simple manner provided the bilinear forms are bounded and the approximation properties of $V_{h}$ and $W_{h}$ are known in these norms. These results can be used to analyze, for example, certain hybrid methods for the biharmonic problem $[5,6]$ and the stationary Stokes problem [10]. The results of $[1,2]$ have also been used to analyze a variety of variationally posed problems that are not of form (1.1). 
There are other problems of a similar nature, however, where attempts at using the ideas of $[1,2,5]$ were not entirely successful since not all of the abstract hypotheses were satisfied: specifically the Brezzi condition (1.3) or, equivalently, the Babuška condition (1.6), is not satisfied with the usual choice of norms, i.e., the approximation methods for these problems are not stable with respect to the usual norms. This is the case, for example, in the analysis in [7] of the HermannMiyoshi $[14,15,19]$ mixed method for the biharmonic problem. In the analysis of this method a natural choice for both $\|.\|_{V}$ and $\|.\|_{W}$ is the 1st order Sobolev norm; however this method is not stable with respect to this choice. As a result of this difficulty, the error estimates obtained in [7] are not quasi-optimal. A similar difficulty arises in the analysis of the Hermann-Johnson $[14,15,16]$ and CiarletRaviart [9] mixed methods for the biharmonic problem. In later work of Scholz [23] and Rannacher [21] quasi-optimal error estimates were obtained for the mixed methods of Ciarlet-Raviart and Hermann-Miyoshi, although the systematic approach of Brezzi and Babuška was abandoned.

In a forthcoming paper of Babuška, Osborn, and Pitkäranta [3] quasi-optimal error estimates for mixed methods for the biharmonic problem are derived by an application of the results of Brezzi and Babuška. In this work a new family of (mesh dependent) norms are introduced with respect to which the above mentioned mixed methods (Ciarlet-Raviart, Hermann-Miyoshi, HermannJohnson) are stable. Error estimates in these norms then follow directly from the results of Brezzi and Babuška, once the approximation properties of the subspaces $V_{h}$ and $W_{h}$ have been determined in these new norms. Error estimates in the more standard norms are then obtained by using the usual duality argument.

It is the intent of this paper to provide an abstract approach to the analysis of mixed methods which leads to quasi-optimal error estimates, uses only standard norms, and is systematic. We shall assume that existence and uniqueness for the continuous (infinite dimensional) problem has been established and develop an abstract framework under which quasi-optimal error estimates can be derived for a variety of examples which do not fit within the convergence theory of Brezzi and Babuska using the usual norms.

Section 2 contains the abstract convergence results of the paper. In section 3 we present four examples previously analyzed in the literature and show how error estimates can be derived from the theorems in section 2. Three of these methods are mixed methods for the biharmonic problem and the fourth is a mixed method for a second order problem analyzed by RaviartThomas $[22,25]$.

It is interesting to note that in this last example the results of Brezzi and Babuska apply with the choice of spaces used by Raviart-Thomas, but fail to 
yield quasi-optimal error estimates in all cases due to the way in which the variables are tied together in the error estimates. In our analysis the error estimates for the two variables are separated and quasi-optimal error estimates are obtained. For the three mixed methods for the biharmonic problem that are analyzed in section 3 the results of the present paper and those obtained in [3], using different techniques, are the same. For additional results on mixed methods see Oden [20]. Finally we note that some basic ideas in the analysis in this paper are similar to those employed in Scholz [23, 24].

Throughout this paper, we shall use the Sobolev spaces $W^{m, p}(\Omega)$, where $\Omega$ is a convex polygon in the plane, $m$ is a nonnegative integer, and $1 \leqq p<\infty$. On these spaces we have the seminorms and norms

and

$$
|v|_{m, p, \Omega}=\left(\int_{\Omega} \sum_{|\alpha|=m}\left|D^{\alpha} v\right|^{p} d x\right)^{1 / p}
$$

$$
\|v\|_{m, p, \Omega}=\left(\int_{\Omega} \sum_{|\alpha| \leqq m}\left|D^{\alpha} v\right|^{p} d x\right)^{1 / p}
$$

When $p=2$, we denote $W^{m, 2}(\Omega)$ by $H^{m}(\Omega)$ and write

and

$$
|v|_{m, 2, \Omega}=|v|_{m, \Omega}
$$

$$
\|v\|_{m, 2, \Omega}=\|v\|_{m, \Omega} \text {. }
$$

We will further denote by $W_{0}^{1, p}(\Omega)$ the subspace of $W^{1, p}(\Omega)$ of functions that vanish on $\Gamma=\partial \Omega$ and by $H_{0}^{2}(\Omega)$ the subspace of $H^{2}(\Omega)$ of functions that vanish together with their normal derivatives on $\Gamma$. For $m=1$ and 2 we will also use the spaces $H^{-m}(\Omega)=\left[H_{0}^{m}(\Omega)\right]^{\prime}$ [the dual space of $\left.H_{0}^{m}(\Omega)\right]$ with the norm on $H^{-m}(\Omega)$ taken to be the usual dual norm. To further simplify notation we often drop the use of the subscript $\Omega$ in the norm when the context is clear.

\section{ABSTRACT RESULTS}

Let $V, W$, and $H$ be three real Banach spaces with norms $\|\cdot\|_{V},\|\cdot\|_{W}$, and $\|.\|_{H}$ respectively. We assume $V \subset H$ with a continuous imbedding. Let $a(.,$. and $b(.,$.$) be continuous bilinear forms on H \times H$ and $V \times W$, respectively:

$$
\begin{gathered}
|a(u, v)| \leqq\|a\| \cdot\|u\|_{H}\|v\|_{H}, \quad \forall u, v \in H, \\
|b(u, \psi)| \leqq\|b\| \cdot\|u\|_{V}\|\psi\|_{W}, \quad \forall u \in V, \quad \forall \psi \in W .
\end{gathered}
$$


We consider the following problem, which we refer to as problem $P$ :

Given $f \in V^{\prime}$ and $g \in W^{\prime}$, find $(u, \psi) \in V \times W$ satisfying:

$$
\begin{gathered}
a(u, v)+b(v, \psi)=(f, v), \quad \forall v \in V, \\
b(u, \varphi)=(g, \varphi), \quad \forall \varphi \in W,
\end{gathered}
$$

where (., .) denotes the pairing between $V$ and $V^{\prime}$ or $W$ and $W^{\prime}$.

We shall be interested in this problem for a subclass of data, i. e., for $(f, g) \in D$, where $D$ is a subclass of $V^{\prime} \times W^{\prime}$. We shall assume that:

(H1) For $(f, g) \in D, P$ has a unique solution.

In the analysis of problem $P$ we will also consider the adjoint problem:

Given $d \in G^{\prime}$, where $G$ is a Banach space satisfying $W \subset G$ with a continuous imbedding, find $(y, \lambda)=\left(y_{d}, \lambda_{d}\right) \in V \times W$ satisfying

$$
\begin{gathered}
a(v, y)+b(v, \lambda)=0, \quad \forall v \in V, \\
b(y, \varphi)=(d, \varphi),-\quad \forall \varphi \in W .
\end{gathered}
$$

We shall assume that:

(H2) Problem (2.5)-(2.6) has a unique solution for each $d \in G^{\prime}$.

Throughout this paper we shall be concerned with the problem of approximating the solution $(u, \psi)$ of $P$. Toward this end, we suppose we are given finite dimensional spaces $V_{h} \subset V$ and $W_{h} \subset W$. We then consider the following approximate problem, which we refer to as problem $P_{h}$ :

Find $\left(u_{i}, \psi_{h}\right) \in V_{h} \times W_{h}$ satisfying:

$$
\begin{gathered}
a\left(u_{h}, v\right)+b\left(v, \psi_{h}\right)=(f, v), \quad \forall v \in V_{h}, \\
b\left(u_{h}, \varphi\right)=(g, \varphi), \quad \forall \varphi \in W_{h} .
\end{gathered}
$$

We will then view $u_{h}$ as an approximation to $u$ and $\psi_{h}$ as an approximation to $\psi$. In this section we obtain estimates for $u-u_{h}$ and $\psi-\psi_{h}$.

We now state several further assumptions which we will require in the proofs of our main results.

(H3) There is a constant $\alpha>0$ ( $\alpha$ independent of $h$ ) such that

$$
a(v, v) \geqq \alpha\|v\|_{H}^{2}, \quad \forall v \in Z_{h},
$$

where $Z_{h}=\left\{v \in V_{n}: b(v, \varphi)=0, \forall \varphi \in W_{h}\right\}$.

(H4) $S(h)$ is a number satisfying

$$
\|v\|_{V} \leqq S(h)\|v\|_{H}, \quad \forall v \in V_{h} .
$$

vol. $14, \mathrm{n}^{\circ} 3,1980$ 
(H5) There is an operator $\pi_{h}: Y \rightarrow V_{h}$ satisfying

$$
b\left(y-\pi_{h} y, \varphi\right)=0, \quad \forall y \in Y \quad \text { and } \forall \varphi \in W_{h},
$$

where $Y=\operatorname{span}\left(\left\{y_{d}\right\}_{d \in G^{\prime}}, u\right),(u, \psi)$ is the solution of problem $P$, and $\left(y_{d}, \lambda_{d}\right)$ is the solution of (2.5)-(2.6) corresponding to $d \in G^{\prime}$.

For the examples treated in section 3 the existence and uniqueness of the approximate solution $\left(u_{h}, \psi_{h}\right)$ can be established in various ways. We now give a proof based on the assumptions made above.

Theorem 1: Assume that hypotheses (H2), (H3) and (H5) are valid. Then problem $P_{h}$ has a unique solution.

Proof: Since $V_{h}$ and $W_{h}$ are finite dimensional, it suffices to show that if $\left(u_{h}, \psi_{h}\right) \in V_{h} \times W_{h}$ satisfies

$$
\begin{gathered}
a\left(u_{h}, v\right)+b\left(v, \psi_{h}\right)=0, \quad \forall v \in V_{h}, \\
b\left(u_{h}, \varphi\right)=0, \quad \forall \varphi \in W_{h},
\end{gathered}
$$

then $u_{h}=\psi_{h}=0$. Choosing $v=u_{h}$ in (2.9) and $\varphi=-\psi_{h}$ in (2.10) and adding the equations, we get $a\left(u_{h}, u_{h}\right)=0$.

Noting from (2.10) that $u_{h} \in Z_{h}$ and using (H3) we have $\left\|u_{h}\right\|_{H}=0$. Hence $u_{h}=0$.

Setting $u_{h}=0$ in (2.9) we obtain:

Now

$$
b\left(v, \psi_{h}\right)=0, \quad \forall v \in V_{h} .
$$

$$
\left\|\psi_{h}\right\|_{G}=\sup _{d \in G^{\prime}} \frac{\left(d, \psi_{h}\right)}{\|d\|_{G^{\prime}}}
$$

By (H2), for each $d \in G^{\prime}$, there exists $y_{d} \in V$ such that for all $\varphi \in W$ :

$$
(d, \varphi)=b\left(y_{d}, \varphi\right)
$$

Thus

$\left(d, \psi_{h}\right)=b\left(y_{d}, \psi_{h}\right)=b\left(\pi_{h} y_{d}, \psi_{h}\right) \quad$ [applying (H5)] $=0 \quad$ [using (2.11)].

Equation (2.12) then implies $\psi_{h}=0$.

Our main result in this section are theorems 2 and 3 which present abstract estimates for the errors $u-u_{h}$ and $\psi-\psi_{h}$. 
THEOREM 2: Suppose hypotheses (H1)-(H5) are valid and that $(u, \psi)$ and $\left(u_{h}, \psi_{h}\right)$ are the respective solutions of problems $P$ and $P_{h}$. Then [with $\pi_{h}$ defined by $\left.(\mathrm{H} 5)\right]$, $\left\|u-u_{h}\right\|_{H} \leqq \frac{1}{\alpha}\left[\|b\| S(h)\|\psi-\varphi\|_{W}\right.$

$$
\left.+(\|a\|+\alpha)\left\|u-\pi_{h} u\right\|_{H}\right] \text { for all } \varphi \in W_{h}
$$

and

$$
\begin{aligned}
\left\|u-u_{h}\right\|_{V} \leqq & \left\|u-\pi_{h} u\right\|_{V}+\frac{S(h)}{\alpha} \\
& \times\left[\|b\| S(h)\|\psi-\varphi\|_{W}+\|a\|\left\|u-\pi_{h} u\right\|_{H}\right] \text { for all } \varphi \in W_{h} .
\end{aligned}
$$

If in addition

$$
Z_{h} \subset Z,
$$

where

$$
Z=\{v \in V: b(v, \varphi)=0, \forall \varphi \in W\}
$$

then

$$
\left\|u-u_{h}\right\|_{H} \leqq\left[1+\frac{\|a\|}{\alpha}\right]\left\|u-\pi_{h} u\right\|_{H}
$$

and

$$
\left\|u-u_{h}\right\|_{V} \leqq\left\|u-\pi_{h} u\right\|_{V}+\frac{S(h)\|a\|}{\alpha}\left\|u-\pi_{h} u\right\|_{H} .
$$

Proof: Using (2.3) we see that

$$
\begin{aligned}
a\left(\pi_{h} u, v\right)+b(v, \psi)=a(u, v)+b(v, \psi) & +a\left(\pi_{h} u-u, v\right) \\
& =(f, v)+a\left(\pi_{h} u-u, v\right), \quad \forall v \in V_{h},
\end{aligned}
$$

and from (2.4) and (H5) we see that

$$
b\left(\pi_{h} u, \varphi\right)=(g, \varphi), \quad \forall \varphi \in W_{h} .
$$

Subtracting (2.7) from (2.17) we find

$$
a\left(\pi_{h} u-u_{h}, v\right)+b\left(v, \psi-\psi_{h}\right)=a\left(\pi_{h} u-u, v\right), \quad \forall v \in V_{h},
$$

and subtracting (2.8) from (2.18) we obtain:

$$
b\left(\pi_{h} u-u_{h}, \varphi\right)=0, \quad \forall \varphi \in W_{h} .
$$

vol. $14, n^{\circ} 3,1980$ 
Choosing $v=\pi_{h} u-u_{h}$ in (2.19) we have

$$
a\left(\pi_{h} u-u_{h}, \pi_{h} u-u_{h}\right)+b\left(\pi_{h} u-u_{h}, \psi-\psi_{h}\right)=a\left(\pi_{h} u-u, \pi_{h} u-u_{h}\right) .
$$

Applying (2.20) we get

$$
\begin{aligned}
& a\left(\pi_{h} u-u_{h}, \pi_{h} u-u_{h}\right)=a\left(\pi_{h} u-u, \pi_{h} u-u_{h}\right) \\
& +b\left(u_{h}-\pi_{h} u, \psi-\varphi\right) \text { for all } \varphi \in W_{h} .
\end{aligned}
$$

Using (2.1), (2.2), (H3), (H4) and noting from (2.20) that $\pi_{h} u-u_{h} \in Z_{h}$, we then obtain:

$$
\begin{aligned}
\alpha\left\|\pi_{h} u-u_{h}\right\|_{H}^{2} \leqq\|a\| \cdot\left\|\pi_{h} u-u\right\|_{H}\left\|\pi_{h} u-u_{h}\right\|_{H} \\
+\|b\| S(h)\left\|u_{h}-\pi_{h} u\right\|_{H}\|\psi-\varphi\|_{W}
\end{aligned}
$$

and hence

$$
\left.\left\|\pi_{h} u-u_{h}\right\|_{H} \leqq \frac{1}{\alpha}\left[\|a\| \cdot\left\|u-\pi_{h} u\right\|_{n}+\|b\| S(h)\|\psi-\varphi\|_{W}\right]\right\}
$$

Thus

$$
\begin{aligned}
\left\|u-u_{h}\right\|_{H} \leqq\left\|u-\pi_{h} u\right\|_{H}+\| & \pi_{h} u-u_{h} \|_{H} \\
& \leqq \frac{1}{\alpha}\left[\|b\| S(h)\|\psi-\varphi\|_{W}+(\|a\|+\alpha)\left\|u-\pi_{h} u\right\|_{H}\right]
\end{aligned}
$$

for all $\varphi \in W_{h}$. This proves (2.13).

In order to prove $(2.14)$ we first note that

$$
\left\|u-u_{h}\right\|_{V} \leqq\left\|u-\pi_{h} u\right\|_{V}+\left\|\pi_{h} u-u_{h}\right\|_{V} \leqq\left\|u-\pi_{h} u\right\|_{V}+S(h)\left\|\pi_{h} u-u_{h}\right\|_{H} .
$$

(2.14) now follows from (2.22).

To prove (2.15) we observe that (2.20) together with $Z_{h} \subset Z$ implies that

$$
b\left(\pi_{h} u-u_{h}, \varphi\right)=0, \quad \forall \varphi \in W .
$$

Hence (2.21) simplifies to

$$
a\left(\pi_{h} u-u_{h}, \pi_{h} u-u_{h}\right)=a\left(\pi_{h} u-u, \pi_{h} u-u_{h}\right) .
$$

Applying (2.1) and (H3) to (2.24) yields

$$
\left\|\pi_{h} u-u_{h}\right\|_{H} \leqq \frac{\|a\|}{\alpha}\left\|\pi_{h} u-u\right\|_{H} .
$$


(2.15) follows by the triangle inequality.

To establish (2.16) we write

$$
\begin{aligned}
& \left\|u-u_{h}\right\|_{V} \leqq\left\|u-\pi_{h} u\right\|_{V}+\left\|\pi_{h} u-u_{h}\right\|_{V} \\
& \quad \leqq\left\|u-\pi_{h} u\right\|_{1}+S(h)\left\|\pi_{h} u-u_{h}\right\|_{H} \quad[\text { by (H4)] } \\
& \quad \leqq\left\|u-\pi_{h} u\right\|_{V}+S(h) \frac{\|a\|}{\alpha}\left\|u-\pi_{h} u\right\|_{H} \quad \text { [using }
\end{aligned}
$$

COROLlary: Inequality (2.15) holds without assumption (H4).

THEOREM 3: (a) Suppose hypotheses $(\mathrm{H} 1),(\mathrm{H} 2),(\mathrm{H} 3)$, and $(\mathrm{H} 5)$ are valid and that $(u, \psi)$ and $\left(u_{h}, \psi_{h}\right)$ are the respective solutions of problems $P$ and $P_{h}$. Then (with $\left(y_{d}, \lambda_{d}\right)$ and $\pi_{h}$ as defined in $(\mathrm{H} 2)$ and $(\mathrm{H} 5)$, respectively),

$$
\begin{aligned}
\left\|\psi-\psi_{h}\right\|_{G}=\sup _{d \in G^{\prime}}\left\{b\left(y_{d}-\pi_{h} y_{d}, \psi-\varphi\right)+a\left(u_{h}-u, \pi_{h} y_{d}-y_{d}\right)\right. & \\
& \left.+b\left(u-u_{k}, \lambda_{d}-\eta\right)\right\} /\|d\|_{G^{\prime}} \text { for all } \bar{\varphi}, \eta \in W_{h}
\end{aligned}
$$

(b) If in addition (H6) holds $\left(Z_{h} \subset Z\right)$, then

$$
b\left(u-u_{h}, \lambda_{d}-\eta\right)=b\left(u-\pi_{h} u, \lambda_{d}-\eta\right), \quad \forall \eta \in W_{h} .
$$

(c) If we further have that:

(H7) There is an operator $\Sigma_{h}: \Lambda \rightarrow W_{h}$ satisfying $b\left(v, \Sigma_{h} \lambda-\lambda\right)=0$ for all $v \in V_{h}$ and all $\lambda \in \Lambda$, where $\Lambda=\operatorname{span}\left(\left\{\lambda_{d}\right\}_{d \in G^{\prime}}, \psi\right),(u, \psi)$ is the solution of problem $P$, and $\left(y_{d}, \lambda_{d}\right)$ is the soiution of (2.5)-(2.6) corresponding to $d \in G^{\prime}$, then

and

$$
b\left(y_{d}-\pi_{h} y_{d}, \psi-\Sigma_{h} \psi\right)=\left(d, \psi-\Sigma_{h} \psi\right)
$$

$$
b\left(u-u_{h}, \lambda_{d}-\Sigma_{h} \lambda_{d}\right)=\left(g, \lambda_{d}-\Sigma_{h} \lambda_{d}\right) .
$$

Proof: From (2.6) we have

$$
\left\|\psi-\psi_{h}\right\|_{G}=\sup _{d \in G^{\prime}}\left(d, \psi-\psi_{h}\right) /\|d\|_{G^{\prime}}=\sup _{d \in G^{\prime}} b\left(y_{d}, \psi-\psi_{h}\right) /\|d\|_{G^{\prime}}
$$

Subtraction of (2.7) from (2.3) and (2.8) from (2.4) yields

$$
a\left(u-u_{h}, v\right)+b\left(v, \psi-\psi_{h}\right)=0, \quad \forall v \in V_{h}
$$

and

$$
b\left(u-u_{h}, \eta\right)=0, \quad \forall \eta \in W_{h} .
$$

vol. $14, n^{\circ} 3,1980$ 
Now, combining (2.5), (H5), (2.31), and (2.32) we obtain:

$$
\begin{gathered}
b\left(y_{d}, \psi-\psi_{h}\right)=b\left(y_{d}-\pi_{h} y_{d}, \psi-\psi_{h}\right)+b\left(\pi_{h} y_{d}, \psi-\psi_{h}\right) \\
=b\left(y_{d}-\pi_{h} y_{d}, \psi-\varphi\right)+a\left(u_{h}-u, \pi_{h} y_{d}\right) \\
=b\left(y_{d}-\pi_{h} y_{d}, \psi-\varphi\right)+a\left(u_{h}-u, \pi_{h} y_{d}-y_{d}\right)+a\left(u_{h}-u, y_{d}\right) \\
=b\left(y_{d}-\pi_{h} y_{d}, \psi-\varphi\right)+a\left(u_{h}-u, \pi_{h} y_{d}-y_{d}\right)+b\left(u-u_{h}, \lambda_{d}\right) \\
=b\left(y_{d}-\pi_{h} y_{d}, \psi-\varphi\right)+a\left(u_{h}-u, \pi_{h} y_{d}-y_{d}\right) \\
+b\left(u-u_{h}, \lambda_{d}-\eta\right) \text { for all } \varphi, \eta \in W_{h} .
\end{gathered}
$$

Substitution of this identity in (2.30) yields $(2.26)$.

If $Z_{n} \subset Z$ then

$$
b\left(\pi_{h} u-u_{h}, \varphi\right)=0, \quad \forall \varphi \in W \quad \text { [see (2.23) above] }
$$

and so (2.27) follows immediately.

Now, if in addition (H7) holds, then

$$
b\left(y_{d}-\pi_{h} y_{d}, \psi-\Sigma_{h} \psi\right)=b\left(y_{d}, \psi-\Sigma_{h} \psi\right)=\left(d, \psi-\Sigma_{h} \psi\right) \quad[\text { by (2.6)]. }
$$

and

$$
b\left(u-u_{h}, \lambda_{d}-\Sigma_{h} \lambda_{d}\right)=b\left(u, \lambda_{d}-\Sigma_{h} \lambda_{d}\right)=\left(g, \lambda_{d}-\Sigma_{h} \lambda_{d}\right) .
$$

Thus (2.28) and (2.29) are established.

REMARK: Note that inequality (2.15) in theorem 2 and all the results of theorem 3 hold without assumption (2.2). This observation is used in subsection $3 c$.

We end this section with several remarks on the hypotheses $(\mathrm{H} 3)-(\mathrm{H} 7)$. We assume here that $V$ and $W$ are Hilbert spaces.

1) It is clear that if

$$
a(v, v) \geqq \gamma_{0}\|v\|_{V}^{2} \text { for all } v \in Z_{h},
$$

then hypotheses (1.3) in Brezzi's theorem is valid. In the applications we consider in section 3, (2.33) is not true (with $\gamma_{0}$ independent of $h$ ) but is valid when $\|v\|_{V}$ is replaced by $\|v\|_{H}$. This accounts for (H3) [and (H4)].

2) In hypotheses (H5)-(H7) it appears that we are not making use of conditions similar to (1.4). In fact, in applications the operator $\pi_{h}$ described in (H5) is often constructed in order to verify (1.4). A more precise relationship is given below in propositions 1 and 2. For further ideas in this direction, consult the work of Fortin [11]. 
Proposition 1: Suppose

$$
\sup _{v \in V_{h}} \frac{|b(v, \varphi)|}{\|v\|_{V}} \geqq k_{0}\|\varphi\|_{W}, \quad \forall \varphi \in W_{h} \text { and } \forall h,
$$

where $k_{0}>0$. Then there is an operator $\pi_{h}: V \rightarrow V_{h}$ that satisfies

$$
b\left(v-\pi_{h} v, \varphi\right)=0, \quad \forall v \in V \text { and } \forall \varphi \in W_{h}
$$

and

$$
\left\|\pi_{h} v\right\|_{V} \leqq \frac{\|b\|}{k_{0}}\|v\|_{V}, \quad \forall v \in V
$$

Proof: Consider $b(v, \varphi)$ on $Z_{h}^{\perp} \times W_{h}$, where $Z_{h}^{1}=\left\{v \in V_{h}: v\right.$ is $V$-orthogonal to $\left.Z_{h}\right\}$. We immediately see that

and

$$
\sup _{v \in Z_{h}^{\perp}} \frac{|b(v, \varphi)|}{\|v\|_{V}} \geqq k_{0}\|\varphi\|_{W}, \quad-\forall \varphi \in W_{h}
$$

$$
\sup _{\varphi \in W_{h}}|b(v, \varphi)|>0, \quad \forall 0 \neq v \in Z_{h}^{\perp} .
$$

It thus follows from $[1,2]$ that for each $v \in V$ there is a unique $\pi_{h} v \in Z_{h}^{\perp}$ satisfying

$$
b\left(\pi_{h} v, \varphi\right)=b(v, \varphi), \quad \forall \varphi \in W_{h} .
$$

Furthermore,

$$
\left\|\pi_{h} \mathrm{v}\right\|_{V} \leqq \frac{\|b\|}{k_{0}}\|v\|_{V}
$$

This proves proposition 1 .

We also note that it follows from $[1,2]$ that for each $\varphi \in W$ there exists a unique $\Sigma_{h} \varphi \in W_{h}$ satisfying

Furthermore,

$$
b\left(v, \Sigma_{h} \varphi\right)=b(v, \varphi), \quad \forall v \in Z_{h}^{\perp} .
$$

$$
\left\|\Sigma_{h} \varphi\right\|_{W} \leqq \frac{\|b\|}{k_{0}}\|\varphi\|_{W}
$$

Proposition 2: Suppose

$$
\sup _{\imath \in V} \frac{|b(v, \varphi)|}{\|v\|_{V}} \geqq k\|\varphi\|_{W}, \quad \forall \varphi \in W,
$$


where $k>0$, and suppose there is an operator $\pi_{h}: V \rightarrow V_{h}$ satisfying

$$
b\left(v-\pi_{h} v, \varphi\right)=0, \quad \forall \varphi \in W_{h}
$$

and

$$
\left\|\pi_{h} v\right\|_{V} \leqq C\|v\|_{V}, \quad \forall v \in V .
$$

Then (2.34) holds.

Proof: Clearly we have

$$
\begin{aligned}
& \sup _{v \in V_{h}} \frac{|b(v, \varphi)|}{\|v\|_{V}} \geqq \sup _{v \in V} \frac{\left|b\left(\pi_{h} v, \varphi\right)\right|}{\left\|\pi_{h} v\right\|_{V}} \\
&=\sup _{v \in V} \frac{|b(v, \varphi)|}{\|v\|_{V}} \frac{\|v\|_{V}}{\left\|\pi_{h} v\right\|_{V}} \geqq \frac{k}{C}\|\varphi\|_{W}, \quad \forall \varphi \in W_{h},
\end{aligned}
$$

i. e., (2.34) holds with $k_{0}=k / C$.

Thus we see that (H5) is closely related to (2.34), which is the same as (1.4).

3) Hypotheses (H6) and (H7) are also closed related as we see by the following result.

Proposition 3: $\Sigma_{h}: W \rightarrow W_{h}$, as defined in remark 2, satisfies

$$
b\left(v, \Sigma_{h} \varphi\right)=h(v, \varphi), \quad \forall v \in V_{h} .
$$

if and only if $Z_{h} \subset Z$.

Proof: Suppose (2.36) holds. Let $v \in Z_{h}$. Then

i.e., $v \in Z$. Thus $Z_{k} \subset Z$.

$$
h(v, \varphi)=b\left(v, \Sigma_{h} \varphi\right)=0, \quad \forall \varphi \in W,
$$

Now suppose $Z_{n} \subset Z$. Then, if $v \in Z_{h}^{\frac{1}{h}}$ we have (2.36) by the definition of $\Sigma_{h}$, and, if $v \in Z_{h}$ we have (2.36) since both terms are zero. Since $V_{h}=Z_{n} \oplus Z_{h}^{\perp}$ we obtain (2.36) for all $v \in V_{h}$.

\section{APPLICATIONS}

In this section we apply the results of section 2 to several examples.

\section{a) Ciarlet-Raviart method}

Consider the biharmonic problem

$$
\left.\begin{array}{cc}
\Delta^{2} \psi=g & \text { in } \Omega, \\
\psi=\frac{\partial \psi}{\partial n}=0 & \text { on } \Gamma=\partial \Omega,
\end{array}\right\}
$$


where $\Omega$ is a convex polygon and $g$ is a given function. If $g \in H^{-2}(\Omega)$ then there is a unique solution $\psi \in H_{0}^{2}(\Omega)$ of (3.1). In addition the following regularity result is known for this problem: If $g \in H^{-1}(\Omega)$, then $\psi \in H^{3}(\Omega) \cap H_{0}^{2}(\Omega)$ and there is a constant $C$ such that

$$
\|\psi\|_{3} \leqq C\|g\|_{-1}, \quad \forall g \in H^{-1}(\Omega) .
$$

Using the well-known correspondence between the biharmonic problem and the Stokes problem, this regularity result can be deduced from the regularity result for the Stokes problem proved in [17] ( $c f$. also [13]).

We now seek an approximation to the solution $\psi$ of (3.1) by a mixed method, i.e., we introduce an auxiliary variable $(u \equiv-\Delta \psi$ for the method of this subsection), write (3.1) as a lower order system, cast this system in variational form, and then consider the Ritz-Galerkin method corresponding to this variational formulation. In particular, the mixed method we study will be based on the following variational formulation of (3.1), first considered by Glowinski [12] and Mercier [18] and further developed by Ciarlet and Raviart [9]:

Given $g \in H^{-1}(\Omega)$, find $(u, \psi) \in H^{1}(\Omega) \times H_{0}^{1}(\Omega)$ satisfying

$$
\left.\begin{array}{cc}
\int_{\Omega} u v d x-\int_{\Omega} \nabla v \cdot \nabla \psi d x=0, & \forall v \in H^{1}(\Omega), \\
-\int_{\Omega} \nabla u \cdot \nabla \varphi d x=-\int_{\Omega} g \varphi d x, & \forall \varphi \in H_{0}^{1}(\Omega) .
\end{array}\right\}
$$

Using the regularity result (3.2) it is not difficult to show (see theorem 1 of [9]) that if $\psi$ is the solution to (3.1) and $u=-\Delta \psi$, then $(u, \psi)$ is a solution of (3.3), and if $(u, \psi)$ is a solution of (3.3), then $\psi$ is a solution of (3.1) and $u=-\Delta \psi$.

It is clear that (3.3) is an example of problem $P$ of section 2 with

$$
\begin{aligned}
V & =H^{1}(\Omega), \quad W=H_{0}^{1}(\Omega), \quad H=L_{2}(\Omega), \\
a(u, v) & =\int_{\Omega} u v d x \quad \text { and } \quad b(u, \psi)=-\int_{\Omega} \nabla u \cdot \nabla \psi d x
\end{aligned}
$$

(and with $g$ replaced by $-g$ ). Here the subclass $D$ of data for which $(\mathrm{H} 1)$ is satisfied is given by $D=0 \times W^{\prime}$. Since the form a is symmetric, the adjoint problem (2.5), (2.6), with $G=W=H_{0}^{1}(\Omega)$, is the same as problem $P$ and thus is uniquely solvable for all $d \in W^{\prime}$. Hence (H2) is satisfied. Using (3.2) we also have

$$
\left\|y_{d}\right\|_{1}+\left\|\lambda_{d}\right\|_{3} \leqq C\|d\|_{-1} .
$$

Next we discuss the finite dimensional subspaces used in the approximation scheme. For $0<h<1$, let $\tau_{h}$ be a triangulation of $\bar{\Omega}$ with triangles $T$ of diameter vol. $14, \mathrm{n}^{\circ} 3,1980$ 
less than or equal to $h$. We assume the family $\left\{\tau_{h}\right\}$ satisfies the minimal angle condition, i.e., there is a constant $\sigma>0$ such that

$$
\max _{T \in \tau_{h}} \frac{h_{T}}{\rho_{T}} \leqq \sigma, \quad \forall h,
$$

where $h_{T}$ is the diameter of $T$ and $\rho_{T}$ is the diameter of the largest circle contained in $T$, and is quasi-uniform, i. e., there is a constant $\tau>0$ such that

$$
\frac{\max _{T} h_{T}}{\min _{T} h_{T}} \leqq \tau, \quad \forall h .
$$

For $k \geqq 1$ a fixed integer we define

$$
S_{h}=\left\{v \in C^{0}(\bar{\Omega}):\left.v\right|_{T} \in P_{k}, \forall T \in \tau_{h}\right\},
$$

where $P_{k}$ is the space of polynomials of degree $k$ or less in the variables $x_{1}$ and $x_{2}$. We then consider the approximate problem $P_{h}$ with $V_{h}=S_{h}$ and $W_{h}=S_{h} \cap H_{0}^{1}(\Omega)$. Note that this scheme yields direct approximations to $\psi$ and $u=-\Delta \psi$ (the stream function and vorticity in hydrodynamical problems).

To apply our theorems we must check that hypotheses (H3)-(H5) are valid. (H3) is clearly valid with $\alpha=1$ and since our family of triangulations is quasiuniform, (H4) is satisfied with $S(h)=C / h$ for some constant $C$. It remains to check (H5). For $v \in H^{1}(\Omega)$ define $\pi_{h} v$ by:

$$
\begin{gathered}
\pi_{h} v \in V_{h}, \\
\int_{\Omega} \nabla\left(\pi_{h} v\right) \cdot \nabla \varphi d x=\int_{\Omega} \nabla v \cdot \nabla \varphi d x, \quad \forall \varphi \in V_{h}
\end{gathered}
$$

and

$$
\int_{\Omega} \pi_{h} v d x=\int_{\Omega} v d x, \text { i.e., }
$$

let $\pi_{h} v$ be the Neumann projection of $v$ into $V_{h}$. Then (H5) is satisfied, and in addition standard approximability results imply that if $v \in H^{r-2}(\Omega), r \geqq 3$, then

$$
\left.\begin{array}{c}
\left\|v-\pi_{h} v\right\|_{j} \leqq C h^{l-j}\|v\|_{l}, \\
j=0,1 \text { and } 1 \leqq l \leqq \min (k+1, r-2) .
\end{array}\right\}
$$

We are now ready to apply theorems 2 and 3 . Suppose $\psi \in H^{r}(\Omega), r \geqq 3$ and that $k \geqq 2$. Then, using (2.13), (3.6), and standard approximability results, we have 


$$
\begin{aligned}
&\left\|u-u_{h}\right\|_{0} \leqq C\left(h^{-1} \inf _{\varphi \in W_{n}}\|\psi-\varphi\|_{1}+\left\|u-\pi_{h} u\right\|_{0}\right) \\
& \leqq C\left(h^{-1} h^{s-1}\|\psi\|_{s}+h^{s-2}\|u\|_{s-2}\right) \\
&\left.\quad \leqq C h^{s-2}\|\psi\|_{s} \text { (since } u=-\Delta \psi\right),
\end{aligned}
$$

where $s=\min (r, k+1)$.

From (2.14) we find in a similar fashion that

$$
\left\|u-u_{h}\right\|_{1} \leqq C h^{s-3}\|\psi\|_{s}
$$

where $s=\min (r, k+1)$.

Finally, from (2.1), (2.2), (2.26), (3.4), (3.7), and (3.8), we have

$$
\begin{aligned}
\left\|\psi-\psi_{h}\right\|{ }_{1} \leqq C \sup _{d \in H^{-1}(\Omega)}\left\{\left\|y_{d}-\pi_{h} y_{d}\right\|_{1} \inf _{\varphi \in W_{h}}\|\psi-\varphi\|_{1}\right. \\
\left.+\left\|u-u_{h}\right\|_{0}\left\|y_{d}-\pi_{h} y_{d}\right\|_{0}+\left\|u-u_{h}\right\|_{1} \inf _{n \in W_{h}}\left\|\lambda_{d}-\eta\right\|_{1}\right\} /\|d\|_{-1} \\
\quad \leqq C \sup _{d \in H^{-1}(\Omega)}\left\{\left\|y_{d}\right\|_{1} h^{s-1}\|\psi\|_{s}+h^{s-2}\|\psi\|_{s} h\left\|y_{d}\right\|_{1}\right. \\
\left.\quad+h^{s-3}\|\psi\|_{s} h^{2}\left\|\lambda_{d}\right\|_{3}\right\} /\|d\|_{-1} \leqq C h^{s-1}\|\psi\|_{s},
\end{aligned}
$$

where $s=\min (r, k+1)$.

Since (3.7)-(3.9) are valid only for $k \geqq 2$, the methods of this paper do not yield error estimates for the case $k=1$ in this example. For this case the reader is referred to Scholz [24]. The estimates (3.7)-(3.9) improve on those in Ciarlet and Raviart [9]. Scholz [23] obtained (3.7) under the assumption that $\Gamma$ is smooth. (3.7) and (3.9) were obtained by Babuška, Osborn, and Pitkäranta [3].

We remark that theorem 3 could also be used to obtain an error estimate for $\left\|\psi-\psi_{h}\right\|_{0}$ [by choosing $G=L_{2}(\Omega)$ ] when $\psi \in H^{4}(\Omega)$. However in order to get quasi-optimal results we would require the regularity result that $d \in L_{2}(\Omega)$ implies $\lambda_{d} \in \mathrm{H}^{4}(\Omega)$, which is not valid on a convex polygon.

\section{b) Hermann-Miyoshi method}

We consider in this subsection another mixed method for the approximate solution of (3.1). In this method the auxiliary variable is the vector of second partial derivatives of $\psi$.

Let

$$
V=\left\{\mathbf{v}=\left(v_{i j}\right), 1 \leqq i, j \leqq 2: v_{12}=v_{21}, v_{i j} \in H^{1}(\Omega)\right\}
$$

(with the usual product norm), and

$$
W=H_{0}^{1}(\Omega) .
$$

vol. $14, n^{\circ} 3,1980$ 
Then the mixed method we study will be based on the following variational formulation of (3.1).

Given $g \in H^{-1}(\Omega)$, find $(\mathbf{u}, \psi) \in V \times W$ satisfying

$$
\left.\begin{array}{c}
\sum_{\imath, j=1}^{2} \int_{\Omega} u_{\imath \jmath} v_{\imath \jmath} d x+\sum_{\substack{\imath, j=1 \\
\forall \mathbf{v} \in V,}}^{2} \frac{\partial v_{\imath \jmath}}{\partial x_{j}} \frac{\partial \psi}{\partial x_{\imath}} d x=0, \\
\sum_{\imath, j=1}^{2} \int_{\Omega} \frac{\partial u_{\imath \jmath}}{\partial x_{j}} \frac{\partial \varphi}{\partial x_{\imath}} d x=-\int_{\Omega} g \varphi d x, \quad \forall \varphi \in W .
\end{array}\right\}
$$

Using the regularity result (3.2) it is not difficult to show that if $\psi$ is a solution of (3.1) and $\mathbf{u}=\left(u_{\imath \jmath}\right)$ is defined by $u_{\imath \jmath}=\partial^{2} \psi / \partial x_{\imath} \partial x_{\jmath}$, then $(\mathbf{u}, \psi)$ is a solution of (3.10), and if $(\mathbf{u}, \psi)$ is a solution of $(3.10)$, then $\psi$ is a solution of (3.1) and $u_{\imath \jmath}=\partial^{2} \psi / \partial x_{\imath} \partial x_{\jmath}$.

We easily observe that (3.10) is an example of problem $P$ with $V$ and $W$ as above,

$$
H=\left\{\mathbf{v}=\left(v_{\imath \jmath}\right), 1 \leqq l, j \leqq 2: v_{12}=v_{21}, v_{\imath \jmath} \in L_{2}(\Omega)\right\}
$$

(with the usual product norm),

and

$$
a(\mathbf{u}, \mathbf{v})=\sum_{\imath, j=1}^{2} \int_{\Omega} u_{\imath \jmath} v_{\imath \jmath} d x
$$

$$
b(\mathbf{u}, \psi)=\sum_{\imath, j=1}^{2} \int_{\Omega} \frac{\partial u_{\imath \jmath}}{\partial x_{J}} \frac{\partial \psi}{\partial x_{\imath}} d x .
$$

As in subsection $3 a$ the subclass $D$ of data for which (H1) is satisfied is given by $D=0 \times W^{\prime}$, and since a is again symmetric (H2) is satisfied with $G=W=H_{0}^{1}(\Omega)$.

Letting $S_{h}$ be as defined in (3.5), we then consider the approximate problem $P_{h}$ with

$$
V_{h}=\left\{\mathbf{v}=\left(v_{\imath \jmath}\right): v_{12}=v_{21}, v_{\imath \jmath} \in S_{h}\right\}
$$

and

$$
W_{h}=S_{h} \cap H_{0}^{1}(\Omega) .
$$

With this choice for the forms $a$ and $b$ and the spaces $V_{h}$ and $W_{h}$, problem $P_{h}$ now describes the Hermann-Miyoshi method [14, 15, 19] for the approximation of the biharmonic problem. Note that with this method we obtain direct approximations to $\psi$ and $\partial^{2} \psi / \partial \mathrm{x}_{\imath} \partial \mathrm{x}_{j}$ (the displacement and the moments in elasticity problems). 
As in subsection $3 a$ we have hypothesis (H3) satisfied with $\alpha=1$ and (H4) satisfied with $S(h)=C / h$ for some constant $C$. (H5) for this example is contained in lemma 2 in [7]. Moreover, by a minor modification of the proof of lemma 2 in [7] we obtain the existence of $\pi_{h}: V \rightarrow V_{h}$ satisfying:

$$
b\left(\mathbf{v}-\pi_{h} \mathbf{v}, \varphi\right)=0, \quad \forall \varphi \in W_{h}
$$

and for $\mathbf{v} \in V \cap\left[H^{r-2}(\Omega)\right]^{4}, r \geqq 3$, the estimate

and

$$
\left\|\mathbf{v}-\pi_{h} \mathbf{v}\right\|_{j} \leqq C h^{l-j}\|\mathbf{v}\|_{l}, \quad j=0,1
$$

$$
1 \leqq 1 \leqq \min (k+1, r-2) \text {. }
$$

We can now apply theorems 2 and 3 in the same way as in subsection $3 a$. Combining these theorems with (3.11) and standard approximability results, we obtain for $\psi \in H^{r}(\Omega)$, with $r \geqq 3$ and $k \geqq 2$ :

$$
\begin{gathered}
\left\|\mathbf{u}-\mathbf{u}_{h}\right\|_{0} \leqq C h^{s-2}\|\psi\|_{s}, \\
\left\|\mathbf{u}-\mathbf{u}_{h}\right\|_{1} \leqq C h^{s-3}\|\psi\|_{s}
\end{gathered}
$$

and

$$
\left\|\psi-\psi_{h}\right\|_{1} \leqq C h^{s-1}\|\psi\|_{s},
$$

where $s=\min (r, k+1)$. Estimates (3.12)-(3.14) improve those in Brezzi-Raviart [7]. Rannacher [21] recently proved these estimates for $k=2$. Babuška, Osborn, and Pitkäranta [3] proved (3.12) and (3.14).

\section{c) Hermann-Johnson method}

We consider here a further mixed method for the solution of (3.1) in which the auxiliary variable is the vector of second partial derivatives of $\psi$, as in section $3 b$.

Given a triangle $T \in \tau_{h}$ and a function $\mathbf{v}=\left(v_{i j}\right)$ with $v_{i j} \in H^{1}(T), 1 \leqq i, j \leqq 2$, and $v_{12}=v_{21}$ we define

añd

$$
M_{v}(\mathrm{v})=\sum_{i, j=1}^{2} v_{i j} v_{j} v_{i}
$$

$$
M_{\mathrm{v \tau}}(\mathbf{v})=\sum_{i, j=1}^{2} v_{i j} v_{j} \tau_{i}
$$

where $\mathbf{v}=\left(v_{1}, v_{2}\right)$ is the unit outward normal and $\tau=\left(\tau_{1}, \tau_{2}\right)=\left(v_{2},-v_{1}\right)$ is the unit tangent along $\partial T$. Let

$$
\begin{aligned}
V=V(h)=\left\{\mathbf{v}=\left(v_{i j}\right): v_{i j} \in L_{2}(\Omega), v_{12}=v_{21},\right. & \\
& \left.v_{i j}\right|_{T} \in H^{1}(T), \forall T \in \tau_{h},
\end{aligned}
$$

vol. $14, \mathrm{n}^{\circ} 3,1980$ 
and $M_{v}(v)$ is continuous at the interelement boundaries

$$
\text { with }\|\mathbf{v}\|_{V}^{2}=\sum_{i, j=1}^{2} \sum_{T \in \tau_{h}}\left\|v_{\imath \jmath}\right\|_{1, T}^{2}
$$

and

$$
W=W_{0}^{1, p}(\Omega),
$$

where $p$ is some number larger than 2 .

The mixed method we study in this subsection will be based on the following variational formulation of (3.1).

Given $g \in H^{-1}(\Omega)$, find $(u, \psi) \in V \times W$ satisfying

$$
\begin{gathered}
\sum_{\imath, j=1}^{2} \int_{\Omega} u_{\imath \jmath} v_{\imath \jmath} d x+\sum_{T \in \tau_{h}}\left\{\sum_{i, j=1}^{2} \int_{T} \frac{\partial v_{\imath \jmath}}{\partial x_{j}} \frac{\partial \psi}{\partial x_{\imath}} d x-\int_{i T} M_{\mathrm{v \tau}}(\mathbf{v}) \frac{\partial \psi}{\partial \tau} d s\right\}=0, \\
\sum_{T \in \tau_{h}}\left\{\sum_{i, j=1} \int_{T} \frac{\partial u_{\imath}}{\partial x_{j}} \frac{\partial \psi}{\partial x_{\imath}} d x\right. \\
\left.-\int_{\partial T} M_{\mathrm{v \tau}}(\mathbf{u}) \frac{\partial \varphi}{\partial \tau} d s\right\}=-\int_{\Omega} g \varphi d x, \quad \forall \varphi \in W .
\end{gathered}
$$

The correspondence between (3.1) and (3.15) is the same as the correspondence between (3.1) and (3.10), i. e., if $\psi$ is the solution of (3.1), then $\left(\left[\partial^{2} \psi / \partial x_{\imath} \partial x_{j}\right], \psi\right)$ is a solution of (3.15), and if $\left(\left(u_{1}\right), \psi\right)$ is a solution of $(3.15)$, then $\psi$ is the solution of ( 31$)$ and $u_{u}=\hat{\imath}^{2} \psi / \hat{c} r_{i} \hat{c} x_{,}$.

One easily sees that (3.15) is an example of problem $P$ with $V$ and $W$ as above, $H$ as in section $3 b$,

and

$$
a(\mathbf{u}, \mathbf{v})=\sum_{\imath, j=1}^{2} \int_{\Omega} u_{\imath \jmath} v_{\imath \jmath} d x
$$

$$
b(\mathbf{u}, \psi)=\sum_{T \in \tau_{n}}\left\{\sum_{\imath, j=1}^{2} \int_{T} \frac{\partial u_{\imath \jmath}}{\partial x_{j}} \frac{\partial \psi}{\partial x_{\imath}} d x-\int_{\partial T} M_{v \tau}(\mathbf{u}) \frac{\partial \psi}{\partial \tau} d s\right\} .
$$

As in the previous subsections, a subclass $D$ of data for which (H1) is satisfied is given by $D=0 \times H^{-1}(\Omega)$, and since a is again symmetric, (H2) is satisfied for $G=H_{0}^{1}(\Omega)$. We note that in this example the space $V=V(h)$ depends on $h$. For each $h$ the form $b(\mathbf{u}, \psi)$ is bounded on $V(h) \times W\left(\right.$ where $W=W_{0}^{1, p}, p>2$ ) with a bound $b$ that depends on $h$. In the error estimates in this subsection we do not require that this bound be independent of $h$. $C f$. the remark following theorem 3 . 
Letting $S_{h}$ be as defined by (3.5), we then consider the approximate problem $P_{h}$ with

and

$$
V_{h}=\left\{\mathbf{v} \in V:\left.v_{i j}\right|_{T} \in P_{k-1}, \forall T \in \tau_{h}\right\}
$$

$$
W_{h}=S_{h} \cap H_{0}^{1}(\Omega)
$$

With this choice for the forms $a$ and $b$ and the spaces $V_{h}$ and $W_{h}$, we have the method of Hermann-Johnson [14, 15, 16].

As in the previous subsections, hypothesis (H3) is satisfied with $\alpha=1$. We now consider (H5).

For $\mathbf{v} \in V$ we define $\pi_{h} v \in V_{h}$ as in [7], section 4 , i.e., $\pi_{h} \mathbf{v}$ is defined by the conditions

$$
\begin{gathered}
\int_{T^{\prime}} M_{v}\left(\mathbf{v}-\pi_{h} \mathbf{v}\right) q d s=0, \quad \forall q \in P_{k-1} \\
\text { and for all sides } T^{\prime} \text { of } \tau_{h} \\
\int_{T}\left[v_{i j}-\left(\pi_{h} \mathbf{v}\right)_{i j}\right] q d x=0, \quad \forall q \in P_{k-2} \text { and } \forall T \in \tau_{h} .
\end{gathered}
$$

and

By lemma 3 in [7], $\pi_{h} \mathbf{v}$ is uniquely determined by (3.16). Since we can write

$$
b(\mathbf{v}, \mu)=\sum_{T \in \tau_{h}}\left\{-\sum_{i . j=1}^{2} \int_{T} v_{i j} \frac{\partial^{2} \mu}{\partial x_{i} \partial x_{j}} d x+\int_{c T} M_{v}(\mathbf{v}) \frac{\partial \mu}{\partial v} d s\right\},
$$

(3.16) easily implies (H5). We note that by lemma 4 of [7] we also have for all $\mathrm{v} \in V \cap\left[H^{r-2}(\Omega)\right]^{4}, r \geqq 3$, that

$$
\left\|\pi_{h} \mathbf{v}-\mathbf{v}\right\|_{0} \leqq C h^{l}\|\mathbf{v}\|_{l}, \quad 1 \leqq l \leqq \min (k, r-2) .
$$

We next observe that by lemma 5 of [7], $Z_{n} \subset Z$, so that we are in the special cases of theorems 2 and 3. In particular, by the corollary to theorem 2, $(\mathrm{H} 4)$ need not be satisfied in order to apply (2.15), so we shall not require $\left\{\tau_{h}\right\}$ to be quasiuniform. Since we wish to apply theorem 3 , part $c$, we now show that hypothesis (H7) is satisfied. As in the proof of lemma 5 in [7], for $v \in V_{h}$ and $\mu \in W=W_{0}^{1, P}(\Omega)$ we can write

$$
\begin{aligned}
b(\mathbf{v}, \mu)=-\sum_{T \in \tau_{h}} \sum_{i, j=1}^{2} \int_{T} \frac{\partial^{2} v_{i j}}{\partial x_{i} \partial x_{j}} \mu d x & \\
& \quad+\sum_{T^{\prime} \in I_{h}} \int_{T^{\prime}} A\left(T^{\prime}, \mathbf{v}\right) \mu d s+\sum_{a \in J_{h}} B(a, \mathbf{v}) \mu(a),
\end{aligned}
$$

vol. $14, \mathrm{n}^{\circ} 3,1980$ 
where $I_{h}$ is the set of all sides of the triangulation $\tau_{h}, J_{h}$ is the set of all vertices of $\tau_{h}$, and $A\left(T^{\prime}, \mathbf{v}\right)$ is a polynomial of degree less than or equal to $k-2$ in the variable $s$.

For $\mu \in W$ we now choose $\Sigma_{h} \mu \in W_{h}$ so that

$$
\begin{array}{ccc}
\int_{T}\left(\mu-\Sigma_{h} \mu\right) q d x=0, & \forall q \in P_{k-3} \text { and } \quad \forall T \in \tau_{h}, \\
\int_{T^{\prime}}\left(\mu-\Sigma_{h} \mu\right) q d s=0, & \forall q \in P_{k-2} \text { and } \quad \forall T^{\prime} \in I_{h}, \\
\left(\Sigma_{h} \mu-\mu\right)(a)=0, \quad \forall a \in J_{h} . &
\end{array}
$$

The unique solvability of this system is easily checked. Note that by the Sobolev imbedding theorem, $\mu \in W$ implies $\mu \in C^{0}(\bar{\Omega})$. Since for $\mathbf{v} \in V_{h}$ we have $\partial^{2} v_{i j} /\left.\partial x_{i} \partial x_{j}\right|_{T} \in P_{k-3}$ and $A\left(T^{\prime}, v\right) \in P_{k-2}$, it follows from (3.18) that $\Sigma_{h} \mu$, as defined by (3.19)-(3.21), satisfies (H7). Furthermore, by a standard application of the Bramble-Hilbert lemma [4], we obtain for all $\mu \in W \cap H^{r}(\Omega)$ :

$$
\left\|\mu-\Sigma_{h} \mu\right\|_{i} \leqq C h^{l-j}\|\mu\|_{l}, j=0,1 \quad \text { and } \quad 1 \leqq l \leqq \min (r, k+1) .
$$

We are now ready to apply theorems 2 and 3 . Suppose that $k \geqq 1$ and $\psi \in H^{r}(\Omega), r \geqq 3$. From (2.15) and (3.17) we obtain:

$$
\left\|\mathbf{u}-\mathbf{u}_{h}\right\|_{0} \leqq C\left\|\mathbf{u}-\pi_{h} \mathbf{u}\right\|_{0} \leqq C h^{\delta}\|\mathbf{u}\|_{\delta} \leqq C h^{\delta}\|\psi\|_{\delta+2},
$$

where $\delta=\min (k, r-2)$.

To obtain estimates for $\psi-\psi_{h}$ we shall apply theorem 3 in several different ways. Choosing $G=H_{0}^{1}(\Omega), \varphi=\mathscr{I}_{h} \psi$, and $\eta=\mathscr{I}_{h} \lambda_{d}$ (where $\mathscr{I}_{h} \varphi$ denotes the standard Lagrange interpolant of $\varphi$ in $S_{h}$ ), we get from theorem $3(a)-(b)$ that

$$
\begin{aligned}
& \left\|\psi-\psi_{h}\right\|_{1}=\sup _{d \in H^{-1}(\varrho)}\left\{b\left(\mathbf{y}_{d}-\pi_{h} \mathbf{y}_{d}, \psi-\mathscr{I}_{h} \psi\right)\right. \\
& +a\left(\mathbf{u}_{h}-\mathbf{u}, \pi_{h} \mathbf{y}_{d}-\mathbf{y}_{d}\right)+b\left(\mathbf{u}-\pi_{h} \mathbf{u}, \lambda_{d}-\mathscr{I}_{h} \lambda_{d}\right\} /\|d\|{ }_{1} .
\end{aligned}
$$

To estimate the terms in the above expression, we introduce the affine transformation

$$
x=F(\hat{x})=B \hat{x}+b,
$$

mapping the reference triangle $\hat{T}$ with vertices $(0,0),(1,0)$ and $(0,1)$ onto $T$, and set

$$
\hat{\mathbf{v}}(\hat{x})=B^{-1} \mathbf{v} \circ F(\hat{x})\left(B^{-1}\right)^{T},
$$

R.A.I.R.O. Analyse numérique/Numerical Analysis 
where

$$
\hat{\mathbf{v}}=\left(\begin{array}{ll}
\hat{v}_{11} & \hat{v}_{12} \\
\hat{v}_{21} & \hat{v}_{22}
\end{array}\right) \quad \text { and } \quad \mathbf{v}=\left(\begin{array}{ll}
v_{11} & v_{12} \\
v_{21} & v_{22}
\end{array}\right)
$$

and

$$
\hat{\varphi}(\hat{x})=\varphi \circ F(\hat{x}) .
$$

Using the standard change of variables argument, we have that if $\mathbf{v} \in\left[H^{l}(T)\right]^{4}$ and $\varphi \in H^{t}(T)$, where $1 \leqq l$ and $2 \leqq t$, then

$$
\int_{T} \sum_{i, j=1}^{2} \frac{\partial v_{i j}}{\partial x_{j}} \frac{\partial \varphi}{\partial x_{i}} d x=|\operatorname{det} B| \int_{\hat{T}} \sum_{i, j=1}^{2} \frac{\partial \hat{v}_{i j}}{\partial \hat{x}_{j}} \frac{\partial \hat{\varphi}}{\partial \hat{x}_{i}} d \hat{x}
$$

and

$$
\int_{i T} M_{v \tau}(\mathbf{v}) \frac{\partial \varphi}{\partial \tau} d s \leqq \sum_{i=1}^{3}\left\|B^{-1} \tau\right\|^{2}\left\|B^{T} v\right\|\left|T_{i}^{\prime}\right| \int_{\hat{T}_{i}^{\prime}} \hat{\tau}^{T} B^{T} B \hat{v}(\hat{x}) \hat{v} \frac{\partial \hat{\varphi}}{\partial \hat{\tau}} d \hat{s},
$$

where $\hat{\tau}$ and $\hat{v}$ denote the unit tangent and unit outward normal to $\partial \hat{T}$, respectively, $T_{i}^{\prime}$ are the sides of $T$, and $\left|T_{i}^{\prime}\right|=$ length of $T_{i}^{\prime}$.

Since

$$
\|B\| \leqq C h, \quad|\operatorname{det} B| \leqq C h^{2}, \quad\left\|B^{-1}\right\| \leqq C / h
$$

and

$$
\left|T_{i}^{\prime}\right| \leqq C h \quad(c f \cdot[8])
$$

it easily follows that

$$
\begin{aligned}
\left|\int_{T} \sum_{i, j=1}^{2} \frac{\partial v_{i j}}{\partial x_{j}} \frac{\partial \varphi}{\partial x_{i}} d x-\int_{\partial T} M_{\mathrm{v} \tau}(\mathbf{v}) \frac{\partial \varphi}{\partial \tau} d s\right| \\
\leqq C h^{2}\left\{\int_{\hat{T}}\left|\sum_{i, j=1} \frac{\partial \hat{v}_{i j}}{\partial \hat{x}_{j}} \frac{\partial \hat{\varphi}}{\partial \hat{x}_{i}}\right| d \hat{x}+\int_{\partial \hat{T}}\left(\sum_{i, j=1}^{2} \hat{v}_{i j}^{2}\right)^{1 / 2}\left|\frac{\partial \hat{\varphi}}{\partial \hat{\tau}}\right| d s\right\} \\
\leqq C h^{2}\|\hat{\mathbf{v}}\|_{1, \hat{T}}\|\hat{\varphi}\|_{2, \hat{T}} .
\end{aligned}
$$

Now from lemma 4 of [7],

if

$$
\left.\begin{array}{c}
\pi_{h} \mathbf{v}-\hat{\mathbf{v}}=0 \\
\hat{\mathbf{v}} \in P_{k-1}^{4}(\hat{T})=\left\{\hat{\mathbf{v}}=\left(\hat{v}_{i j}\right), \hat{v}_{i j} \in P_{k-1}(\hat{T}), \hat{v}_{12}=\hat{v}_{21}\right\}
\end{array}\right\}
$$

and

$$
\left\|\hat{\pi_{h}} \mathbf{v}-\hat{\mathbf{v}}\right\|_{1, \hat{T}} \leqq C(\hat{T})\|\hat{\mathbf{v}}\|_{1, \hat{T}} .
$$

vol. $14, n^{\circ} 3,1980$ 
Using the standard properties of the interpolant ( $c f$. [8]) we also get

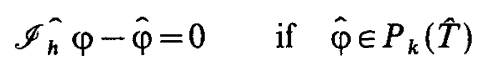

and

$$
\left\|\mathscr{I}_{h} \hat{\varphi}-\hat{\varphi}\right\|_{2, \hat{T}} \leqq C(\hat{T})\|\hat{\varphi}\|_{2, \hat{T}} .
$$

Using (3.24)-(3.28) we easily obtain:

$$
\begin{aligned}
\mid \int_{T} \sum_{i, j=1} \frac{\partial}{\partial x_{j}}\left(\mathbf{v}-\pi_{h} \mathbf{v}\right)_{i j} & \frac{\partial}{\partial x_{i}}\left(\varphi-\mathscr{I}_{h} \varphi\right) d x \\
& \quad-\int_{\partial T} M_{\mathbf{v} \tau}\left(\mathbf{v}-\pi_{h} \mathbf{v}\right) \frac{\partial}{\partial \tau}\left(\varphi-\mathscr{I}_{h} \varphi\right) d s \mid \\
\leqq & C h^{2} \inf _{p \in P_{\hat{k}-1}^{4}(\hat{T})}\|\hat{\mathbf{v}}-p\|_{1, \hat{T}} \inf _{q \in P_{k}(\hat{T})}\|\hat{\varphi}-q\|_{2, \hat{T}} \\
& \leqq C h^{2} \inf _{p \in P_{k-1}^{4}(\hat{T})}\|\hat{\mathbf{v}}-p\|_{l, \hat{T}} \inf _{q \in P_{k}(\hat{T})}\|\hat{\varphi}-q\|_{t, \hat{T}} \\
& \leqq C h^{2}|\hat{\mathbf{v}}|_{l, T}|\hat{\varphi}|_{t, \hat{T}}
\end{aligned}
$$

for $1 \leqq l \leqq k$ and $2 \leqq t \leqq k+1$. Changing back to the original variables we further obtain:

and

$$
|\hat{\mathbf{v}}|_{l, \hat{T}} \leqq C h^{l-3}|\mathbf{v}|_{l, T}
$$

$$
|\hat{\psi}|_{t, \hat{T}} \leqq C h^{t-1}|\psi|_{t, T} .
$$

Hence if $\mathbf{v} \in\left[H^{l}(\Omega)\right]^{4}$ and $\varphi \in H^{t}(\Omega)$ for $1 \leqq l \leqq k$ and $2 \leqq t \leqq k+1$, then

$$
\begin{aligned}
\left|b\left(\mathbf{v}-\pi_{h} \mathbf{v}, \varphi-\mathscr{I}_{h} \varphi\right)\right| & \\
\leqq & \sum_{T} \mid \int_{T i, j=1} \sum_{j}^{2} \frac{\partial}{\partial x_{j}}\left(\mathbf{v}-\pi_{h} \mathbf{v}\right)_{i j} \frac{\partial}{\partial x_{i}}\left(\varphi-\mathscr{I}_{h} \varphi\right) d x \\
& -\int_{\partial T} M_{\mathbf{v}}\left(\mathbf{v}-\pi_{h} v\right) \frac{\partial}{\partial \tau}\left(\varphi-\mathscr{I}_{h} \varphi\right) d s \mid \\
& \leqq \sum_{T} C h^{t+l-2}|\mathbf{v}|_{l, T}|\psi|_{t, T} \leqq C h^{t+l-2}\|\mathbf{v}\|_{l}\|\psi\|_{t} .
\end{aligned}
$$

Choosing $\mathbf{v}=\mathbf{y}_{d}, \varphi=\psi, l=1$ and $t=\min (r, k+1) \equiv s$ in (3.29) we get

$$
\left|b\left(\mathbf{y}_{d}-\pi_{h} \mathbf{y}_{d}, \psi-\mathscr{I}_{h} \psi\right)\right| \leqq C h^{s-1}\left\|\mathbf{y}_{d}\right\|_{1}\|\psi\|_{s} \text {. }
$$

If $k \geqq 2$ we choose $\mathbf{v}=\mathbf{u}, \varphi=\lambda_{d}, l=s-2$, and $t=3$ in (3.29) to obtain:

$$
\left|b\left(\mathbf{u}-\pi_{h} \mathbf{u}, \lambda_{d}-\mathscr{I}_{h} \lambda_{d}\right)\right| \leqq C h^{s-1}\|\mathbf{u}\|_{s-2}\left\|\lambda_{d}\right\|_{3} \leqq C h^{s-1}\|\psi\|_{s}\left\|\lambda_{d}\right\|_{3} .
$$

R.A.I.R.O. Analyse numérique/Numerical Analysis 
If $k=1$ we choose $l=1$ and $t=2$ to obtain:

$$
\left|b\left(\mathbf{u}-\pi_{h} \mathbf{u}, \lambda_{d}-\mathscr{I}_{h} \lambda_{d}\right)\right| \leqq C h\|\mathbf{u}\|{ }_{1}\left\|\lambda_{d}\right\|_{2} \leqq C h\|\psi\|_{3}\left\|\lambda_{d}\right\|_{3} .
$$

Finally if $k \geqq 2$, from (2.1), (2.15), and (3.17) with $l=1$ and $l=s-2$ we have

$$
\begin{aligned}
& \mid a\left(\mathbf{u}_{h}-\mathbf{u}, \pi_{h} \mathbf{y}_{d}-\mathbf{y}_{d}\right) \leqq C\left\|\mathbf{u}_{h}-\mathbf{u}\right\|_{0}\left\|\pi_{h} \mathbf{y}_{d}-\mathbf{y}_{d}\right\|_{0} \\
& \qquad C\left\|\pi_{h} \mathbf{u}-\mathbf{u}\right\|_{0}\left\|\pi_{h} \mathbf{y}_{d}-\mathbf{y}_{d}\right\|_{0} \leqq C h^{s-2}\|\mathbf{u}\|_{s-2} h\left\|\mathbf{y}_{d}\right\|_{1} \\
& \quad \leqq C h^{s-1}\|\psi\|\left\|_{s}\right\| \mathbf{y}_{d} \|_{1} .
\end{aligned}
$$

If $k=1$ we choose $l=1$ in (3.17) to get

$$
\mid a\left(\mathbf{u}_{h}-\mathbf{u}, \pi_{h} \mathbf{y}_{d}-\mathbf{y}_{d}\right) \leqq C h\|\mathbf{u}\|_{1} h\left\|\mathbf{y}_{d}\right\|_{1} \leqq C h^{2}\|\psi\|_{3}\left\|\mathbf{y}_{d}\right\|_{1} .
$$

Applying the regularity result

$$
\left\|\mathbf{y}_{d}\right\|_{1}+\left\|\lambda_{d}\right\|_{3} \leqq C\|d\|_{-1}
$$

and collecting terms, we get

$\left\|\psi-\psi_{h}\right\|_{1} \leqq C h^{s-1}\|\psi\|_{s} \quad$ for $\quad k \geqq 2 \quad$ where $s=\min (r, k+1)(3.31)$ and

$$
\left\|\psi-\psi_{h}\right\|_{1} \leqq C h\|\psi\|_{3} \quad \text { for } \quad k=1 .
$$

We now derive estimates in $L_{2}(\Omega)$. First consider the case when $k=1$ and $\psi \in H^{4}(\Omega)$. Using theorem $3(a)$ - $(c)$ with $G=L_{2}(\Omega), \varphi=\Sigma_{h} \psi$, and $\eta=\Sigma_{h} \lambda_{d}$, and (2.1) we easily obtain:

$$
\begin{aligned}
& \begin{array}{l}
\left\|\psi-\psi_{h}\right\|_{0} \leqq C \sup _{d \in L_{2}(\Omega)}\left\{\|d\|_{0}\left\|\psi-\Sigma_{h} \psi\right\|_{0}+\left\|\mathbf{u}-\mathbf{u}_{h}\right\|_{0}\left\|\mathbf{y}_{d}-\pi_{h} \mathbf{y}_{d}\right\|_{0}\right. \\
+ \\
+
\end{array} \\
& \text { From (3.22) we get }
\end{aligned}
$$

$$
\left\|\psi-\Sigma_{h} \psi\right\|_{0} \leqq C h^{2}\|\psi\|_{2}
$$

and

$$
\left\|\lambda_{d}-\Sigma_{h} \lambda_{d}\right\|_{0} \leqq C h^{2}\left\|\lambda_{d}\right\|_{2} .
$$

Using (2.15) and (3.17) we have

$$
\left\|\mathbf{u}-\mathbf{u}_{h}\right\|_{0}\left\|\mathbf{y}_{d}-\pi_{h} \mathbf{y}_{d}\right\|_{0} \leqq C\left\|\mathbf{u}-\pi_{h} \mathbf{u}\right\|_{0}\left\|\mathbf{y}_{d}-\pi_{h} \mathbf{y}_{d}\right\|_{0} \leqq C h^{2}\|\mathbf{u}\|_{1}\left\|\mathbf{y}_{d}\right\|_{1} .
$$

Noting that $\|d\|_{-1} \leqq\|d\|_{0}$ and $\|g\|_{0} \leqq C\|\psi\|_{4}$, using (3.30) and combining terms we obtain:

for $k=1$.

$$
\left\|\psi-\psi_{h}\right\|_{0} \leqq C h^{2}\|\psi\|_{4}
$$


Next we consider the case $k \geqq 2$. Using theorems $3(a)-(c)$ with $G=L_{2}(\Omega)$, $\varphi=\Sigma_{h} \psi$, and $\eta=\mathscr{I}_{h} \lambda_{d}$, and (2.1) we have

$$
\begin{aligned}
\left\|\psi-\psi_{h}\right\|_{0} \leqq C \sup _{d \in L_{2}(\Omega)}\left\{\|d\|_{0}\left\|\psi-\Sigma_{h} \psi\right\|_{0}\right. & +\left\|\mathbf{u}-\mathbf{u}_{h}\right\|_{0}\left\|\mathbf{y}_{d}-\pi_{h} \mathbf{y}_{d}\right\|_{0} \\
& \left.+\left|b\left(\mathbf{u}-\pi_{h} \mathbf{u}, \lambda_{d}-\mathscr{I}_{h} \lambda_{d}\right)\right|\right\} /\|d\|_{0} .
\end{aligned}
$$

From (3.22) with $l=\bar{s}=\min (r-1, k+1)$ we get

$$
\left\|\psi-\Sigma_{h} \psi\right\|_{0} \leqq C h^{\bar{s}}\|\psi\|_{\bar{s}} .
$$

Using (2.15) and (3.17) with $l=\bar{s}-1$ we see that

$$
\left\|\mathbf{u}-\mathbf{u}_{h}\right\|_{0} \leqq C h^{\bar{s}-1}\|\psi\|_{\bar{s}+1}
$$

and using (3.17) with $l=1$ and (3.30) we obtain:

$$
\left\|\mathbf{y}_{d}-\pi_{h} \mathbf{y}_{d}\right\|_{0} \leqq C h\|d\|_{0} .
$$

Finally from (3.29) with $\mathbf{v}=\mathbf{u}, \varphi=\lambda_{d}, l=\bar{s}-1$, and $t=3$, and (3.31) we see that

$$
\left|b\left(\mathbf{u}-\pi_{h} \mathbf{u}, \lambda_{d}-\mathscr{I}_{h} \lambda_{d}\right)\right| \leqq C h^{\bar{s}}\|\psi\|_{\bar{s}+1}\|d\|_{0} .
$$

Combining these estimates we have

$$
\left\|\psi-\psi_{h}\right\|_{0} \leqq C h^{\bar{s}}\|\psi\|_{\bar{s}+1},
$$

where $\bar{s}=\min (r-1, k+1)$ and $k \geqq 2$.

Note that (3.34) gives an improvement over (3.31) only for $k+1 \leqq r-1$. Estimates (3.31) improve estimates in [7]. Babuška, Osborn, and Pitkäranta [3] have proved (3.23), (3.31)-(3.34).

\section{d) Raviart-Thomas method}

In our final example we study a mixed method for second order elliptic problems introduced by Raviart and Thomas [22, 25]. For $g \in L_{2}(\Omega), \Omega$ a convex polygon in $\mathbb{R}^{2}$, we consider the model problem

$$
\left.\begin{array}{c}
-\Delta \psi=g \text { in } \Omega \\
\psi=0 \text { on } \Gamma .
\end{array}\right\}
$$

Let $\mathbf{H}(\operatorname{div} ; \Omega)=\left\{\mathbf{v} \in\left[L_{2}(\Omega)\right]^{2}: \operatorname{div} \mathbf{v} \in L_{2}(\Omega)\right\}$ with the norm

$$
\|\mathbf{v}\|_{\mathbf{H}(\text { div; } \Omega)}=\left(\|\mathbf{v}\|_{0}^{2}+\|\operatorname{div} \mathbf{v}\|_{0}^{2}\right)^{1 / 2} \text {. }
$$

The mixed method we study is based on the following variational formulation of (3.35). 
Find $(\mathbf{u}, \psi) \in \mathbf{H}(\operatorname{div} ; \Omega) \times L_{2}(\Omega)$ such that

and

$$
\int_{\Omega} \mathbf{u} \cdot \mathbf{v} d x+\int_{\Omega} \psi \operatorname{div} \mathbf{v} d x=0, \quad \forall \mathbf{v} \in H(\operatorname{div} ; \Omega)
$$

$$
\int_{\Omega} \varphi(\operatorname{div} \mathbf{u}+g) d x=0, \quad \forall \varphi \in L_{2}(\Omega) .
$$

In theorem 1 of [22] it is shown that problem (3.36)-(3.37) has a unique solution $(\mathbf{u}, \psi) \in \mathbf{H}(\operatorname{div} ; \Omega) \times L_{2}(\Omega)$, that $\psi$ is the solution of problem (3.35), and $\mathbf{u}=\operatorname{grad} \psi$. In addition the following regularity result is known for this problem:

If $g \in L_{2}(\Omega)$ then $\psi \in H^{2}(\Omega) \cap H_{0}^{1}(\Omega)$ and

$$
\|\psi\|_{2} \leqq C\|g\|_{0}, \quad \forall g \in L_{2}(\Omega) .
$$

One easily sees that (3.36)-(3.37) is an example of problem $P$ with $V=\mathbf{H}(\operatorname{div} ; \boldsymbol{\Omega}), H=W=L_{2}(\boldsymbol{\Omega}),\left[L_{2}(\boldsymbol{\Omega})\right]^{2}$,

$$
a(\mathbf{u}, \mathbf{v})=\int_{\Omega} \mathbf{u} \cdot \mathbf{v} d x \quad \text { and } \quad b(\mathbf{u}, \psi)=\int_{\Omega} \psi \operatorname{div} \mathbf{u} d x
$$

The subclass $D$ of data for which (H1) is satisfied is given by $D=0 \times W^{\prime}$. Since a is symmetric, the adjoint problem (2.5), (2.6) with $G=W=L_{2}(\Omega)$ is the same as problem $P$ and thus is uniquely solvable for all $d \in W^{\prime}$. Hence (H2) is satisfied. Using (3.38) we also see that $\lambda_{d} \in H^{2}(\Omega) \cap H_{0}^{1}(\Omega), \mathbf{y}_{d}=\operatorname{grad} \lambda_{d}$, and

$$
\left\|\mathbf{y}_{d}\right\|_{1}+\left\|\lambda_{d}\right\|_{2} \leqq C\|d\|_{0} .
$$

We now describe the finite dimensional subspaces used in the approximation scheme. Following [22] we begin by introducing the space $\mathbf{Q}$ associated with the unit right triangle $\hat{T}$ in the $(\xi, \eta)$-plane whose vertices are $\hat{a}_{1}=(1,0), \hat{a}_{2}=(0,1)$, $\hat{a}_{3}=(0,0)$. For $k \geqq 0$ an even integer, define $\hat{\mathbf{Q}}$ to be the space of all functions $\hat{\mathbf{q}}$ of the form

$$
\begin{aligned}
& \hat{q}_{1}=\operatorname{pol}_{k}(\xi, \eta)+\alpha_{0} \xi^{k+1}+\alpha_{1} \xi^{k} \eta+\ldots+\alpha_{k / 2} \xi^{k / 2+1} \eta^{k / 2} \\
& \hat{q}_{2}=\operatorname{pol}_{k}(\xi, \eta)+\beta_{0} \eta^{k+1}+\beta_{1} \xi \eta^{k}+\ldots+\beta_{k / 2} \xi^{k / 2} \eta^{k / 2+1}
\end{aligned}
$$

with

$$
\sum_{i=0}^{k / 2}(-1)^{i}\left(\alpha_{i}-\beta_{i}\right)=0,
$$

where $\operatorname{pol}_{k}(\xi, \eta)$ denotes any polynomial of degree $k$ in the two variables $\xi, \eta$. 
For $k \geqq 1$ an odd integer, define $\mathbf{Q}$ to be the space of all functions $\hat{\mathbf{q}}$ of the form

$$
\begin{aligned}
& \hat{q}_{1}=\operatorname{pol}_{k}(\xi, \eta)+\alpha_{0} \xi^{k+1}+\alpha_{1} \xi^{k} \eta+\ldots+\alpha_{(k+1) / 2} \xi^{(k+1) / 2} \eta^{(k+1) / 2}, \\
& \hat{q}_{2}=\operatorname{pol}_{k}(\xi, \eta)+\beta_{0} \eta^{k+1}+\beta_{1} \xi \eta^{k}+\ldots+\beta_{(k+1) / 2} \xi^{(k+1) / 2} \eta^{(k+1) / 2},
\end{aligned}
$$

with

$$
\sum_{i=0}^{(k+1) / 2}(-1)^{i} \alpha_{i}=\sum_{i=0}^{(k+1) / 2}(-1)^{i} \beta_{i}=0 .
$$

Now consider any triangle $T$ in the $\left(x_{1}, x_{2}\right)$-plane whose vertices are denoted by $a_{i}, 1 \leqq i \leqq 3$. Let $F_{T}: \hat{x} \rightarrow F_{T}(\hat{x})=B_{T} \hat{x}+b_{T}, B_{T} \in \mathscr{L}\left(\mathbb{R}^{2}\right), b_{T} \in \mathbb{R}^{2}$ be the unique invertible affine mapping such that $F_{T}\left(\hat{a}_{i}\right)=a_{i}, 1 \leqq i \leqq 3$. With each vector-valued function $\hat{\mathbf{v}}=\left(\hat{v}_{1}, \hat{v}_{2}\right)$ defined on $\hat{T}$, we associate the function $\mathbf{v}$ defined on $T$ by

$$
\mathbf{v}=\frac{1}{J_{T}} B_{T} \hat{\mathbf{v}}_{\circ} F_{T}^{-1}
$$

where

$$
J_{T}=\operatorname{det}\left(B_{T}\right)
$$

For $0<h<1$, assume that $\tau_{h}$ is a triangulation of $\bar{\Omega}$ made up of triangles $T$ whose diameters are less than or equal to $h$ which satisfy the minimal angle condition (see subsection $3 a$ ). We finally consider problem $P_{h}$ with

$$
V_{h}=\left\{\mathbf{v}_{h} \in \mathbf{H}(\operatorname{div} ; \Omega): \forall T \in \tau_{h},\left.\mathbf{v}_{h}\right|_{T} \in \mathbf{Q}_{T}\right\},
$$

where

$$
\mathbf{Q}_{T}=\{\mathbf{v} \in H(\operatorname{div} ; T): \hat{\mathbf{v}} \in \hat{\mathbf{Q}}\}
$$

and

$$
W_{h}=\left\{\varphi_{h} \in L_{2}(\Omega): \forall T \in \tau_{h},\left.\varphi_{h}\right|_{T} \in P_{k}\right\} .
$$

To apply our theorems we must check that the appropriate hypotheses are satisfied. Now (H3) is trivially satisfied with $\alpha=1$. In the proof of theorem 3 of [22] it is essentially shown that there is an operator $\pi_{h}:\left[H^{1}(\Omega)\right]^{2} \rightarrow V_{h}$ satisfying

$$
b\left(v-\pi_{h} v, \varphi\right)=0, \quad \forall \mathbf{v} \in\left[H^{1}(\Omega)\right]^{2} \quad \text { and } \quad \forall \varphi \in W_{h} .
$$

Furthermore, for $\mathbf{v} \in\left[H^{r-1}(\Omega)\right]^{2}, r \geqq 2$, we have

and

$$
\left\|\mathbf{v}-\pi_{h} \mathbf{v}\right\|_{0} \leqq C h^{l}\|\mathbf{v}\|_{l}, \quad 1 \leqq l \leqq \min (r-1, k+1),
$$

$$
\left\|\operatorname{div}\left(\mathbf{v}-\pi_{h} \mathbf{v}\right)\right\|_{0} \leqq C h^{m}\|\operatorname{div} \mathbf{v}\|_{m}, \quad 0 \leqq m \leqq \min (r-2, k+1) .
$$


Using the regularity results (3.38) and (3.39) we easily see that $Y \subset\left[H^{1}(\Omega)\right]^{2}$ so that $(\mathrm{H} 5)$ is valid.

We next observe that for $\mathbf{v}_{h} \in V_{h},\left.\operatorname{div} \mathbf{v}_{h}\right|_{T} \in P_{k}$. Hence $\mathbf{v}_{h} \in Z_{h}$ easily implies $\operatorname{div} \mathbf{v}_{h}=0$ and so $\mathbf{v}_{h} \in Z$. Thus $Z_{h} \subset Z$ and so we are in the special cases of theorems 2 and 3. Again by the corollary to theorem 2, (H4) need not be satisfied in order to apply (2.15), so we shall not require $\left\{\tau^{h}\right\}$ to be quasiuniform.

We are now ready to derive the error estimates. Assume that $\psi \in H^{r}(\Omega), r \geqq 2$. From (2.15) and (3.40) we obtain for $k \geqq 0$ :

$$
\left\|\mathbf{u}-\mathbf{u}_{h}\right\|_{0} \leqq C\left\|\mathbf{u}-\pi_{h} \mathbf{u}\right\|_{0} \leqq C h^{t}\|\mathbf{u}\|_{t} \leqq C h^{t}\|\psi\|_{t+1},
$$

where $t=\min (r-1, k+1)$.

Now applying theorem $3(a)-(b)$ we get

$$
\begin{aligned}
\left\|\psi-\psi_{h}\right\|_{0}=\sup _{d \in L_{2}(\Omega)} & \left\{b\left(\mathbf{y}_{d}-\pi_{h} \mathbf{y}_{d}, \psi-\varphi\right)\right. \\
& \left.+a\left(\mathbf{u}_{h}-\mathbf{u}, \pi_{h} \mathbf{y}_{d}-\mathbf{y}_{d}\right)+b\left(\mathbf{u}-\pi_{h} \mathbf{u}, \lambda_{d}-\eta\right)\right\} /\|d\|_{0}
\end{aligned}
$$

for all $\varphi, \eta \in W_{h}$. Using (3.41) and standard approximability properties of $W_{h}$, we have

$$
\begin{aligned}
& \inf _{\eta \in W_{h}}\left|b\left(\mathbf{y}_{d}-\pi_{h} \mathbf{y}_{d}, \psi-\varphi\right)\right| \leqq\left\|\operatorname{div}\left(\mathbf{y}_{d}-\pi_{h} \mathbf{y}_{d}\right)\right\|_{0} \inf _{\eta \in W_{h}}\|\psi-\varphi\|_{0} \\
& \leqq C\left\|\operatorname{div} \mathbf{y}_{d}\right\|_{0} h^{\mu}\|\psi\|_{\mu},
\end{aligned}
$$

where $\mu=\min (r, k+1)$, and choosing $m=\mu-2$ in (3.32),

$$
\begin{aligned}
& \inf _{\eta \in W_{h}}\left|b\left(\mathbf{u}-\pi_{h} \mathbf{u}, \lambda_{d}-\eta\right)\right| \leqq\left\|\operatorname{div}\left(\mathbf{u}-\pi_{h} \mathbf{u}\right)\right\|_{0} \inf _{\eta \in W_{h}}\left\|\lambda_{d}-\eta\right\|_{0} \\
& \leqq C h^{\mu-2}\|\operatorname{div} \mathbf{u}\|_{\mu-2} h^{2}\left\|\lambda_{d}\right\|_{2},
\end{aligned}
$$

provided $k \geqq 1$.

Using (2.1), (2.15), and (3.40) (with $l=\mu-1$ ) we obtain for $k \geqq 1$ :

$$
\begin{aligned}
\left|a\left(\mathbf{u}_{h}-\mathbf{u}, \pi_{h} \mathbf{y}_{d}-\mathbf{y}_{d}\right)\right| & \leqq\left\|\mathbf{u}_{h}-\mathbf{u}\right\|_{0}\left\|\pi_{h} \mathbf{y}_{d}-\mathbf{y}_{d}\right\|_{0} \\
& \leqq C\left\|\mathbf{u}-\pi_{h} u\right\|_{0}\left\|\pi_{h} \mathbf{y}_{d}-\mathbf{y}_{d}\right\|_{0} \leqq C h^{\mu-1}\|\mathbf{u}\|_{\mu-1} h\left\|\mathbf{y}_{d}\right\|_{1} .
\end{aligned}
$$

Now from (3.43), (3.44), (3.45), (3.46) and the regularity result (3.39) we obtain for $k \geqq 1$ :

$$
\left\|\psi-\psi_{h}\right\|_{0} \leqq h^{\mu}\|\psi\|_{\mu},
$$

where $\mu=\min (r, k+1)$.

vol. $14, \mathrm{n}^{\circ} 3,1980$ 
To obtain an estimate when $k=0$, we choose $m=0$ in (3 41) to obtain

$$
\begin{aligned}
& \operatorname{lnf}_{\eta \in W_{h}}\left|b\left(\mathbf{u}-\pi_{h} \mathbf{u}, \lambda_{d}-\eta\right)\right| \leqq\left\|\operatorname{div}\left(\mathbf{u}-\pi_{h} \mathbf{u}\right)\right\|_{0} \inf _{\eta \in W_{h}}\left\|\lambda_{d}-\eta\right\|_{0} \\
& \leqq C\|\operatorname{div} \mathbf{u}\|_{0} h\left\|\lambda_{d}\right\|_{1}
\end{aligned}
$$

and choose $l=1$ in $(340)$ to obtain in the same manner as in $\left(\begin{array}{ll}46 & 36\end{array}\right)$ that

$$
\begin{aligned}
\left|a\left(\mathbf{u}_{h}-\mathbf{u}, \pi_{h} \mathbf{y}_{d}-\mathbf{y}_{d}\right)\right| \leqq C\left\|\mathbf{u}-\pi_{h} \mathbf{u}\right\|_{0}\left\|\pi_{h} \mathbf{y}_{d}-\mathbf{y}_{d}\right\|_{0} & \\
& \leqq C h\|\mathbf{u}\|_{1} h\left\|\mathbf{y}_{d}\right\|_{1}
\end{aligned}
$$

Combining (3 43), (3 44) with $k=0$, (3 48), (3 49), and the regularity result (3 39 ) we get

$$
\left\|\psi-\psi_{h}\right\|_{0} \leqq C h\|\psi\|_{2}, \quad k=0
$$

We note that estımate ( 342 ) was obtained in [25], IX-3 $22 a$, and that (3 47) gives an improvement over the result in [25], IX-3 $22 a$, in the case where $\psi \in H^{r}(\Omega), \mathbf{u} \in H^{r-1}(\Omega)$, and $2 \leqq r<k+1$

\section{ACKNOWLEDGEMENT}

The second author would like to thank I Babuška and J Pitkaranta for several helpful discussions on mixed methods

\section{REFERENCES}

1 I B BäǓ̌KA, Error-Bounds for Finite Element Method, Numer Math, Vol 16, 1971, pp 322-333

2 I BABUŠKA and A AzIZ, Survey Lectures on the Mathematical Foundations of the Finite Element Method in The Mathematical Foundations of the Fintte Element Method with Application to Partial Differential Equations, A K Azız, Ed, Academic Press, New York, 1973, pp 5-359

3 I BabušKa, J Osborn and J PttKaranta, Analysis of Mixed Methods for 4th Order Elliptzc Equations (to appear)

$4 \mathrm{~J}$ Bramble and S HilberT, Estimation of Linear Functionals on Sobolov Spaces with Application to Fourler Transforms and Spline Interpolation, S I A M J Numer Anal, Vol 13, 1976, pp 185-197

5 F BREzzi, On the Existence, Uniqueness and Approximation of Saddle-Point Problems Arising from Lagrangian Multiplers, R A I R O, Vol 8 R2, 1974, pp 129-151

6 F BREzZi, Sur la methode des elements finis hybrides pour le probleme biharmonique, Numer Math, Vol 24, 1975, pp 103-131

7 F Brezzi and P RAVIART, Mixed Finite Element Methods for 4th Order Elliptic Equations, Topics in Numerical Analysis III, J Miller, Ed, Academic Press, 1978 
8 P Garlet, The Fintte Element Method for Elliptic Problems, North-Holland, 1978

9 P Ciarlet and P Raviart, A Mixed Finite Element Method for the Biharmonic Equation, Symposium on Mathematical Aspects of Fintte Elements in Partial Differential Equations, C DE Boor, Ed, Academic Press, New York, 1974, pp 125143

$10 \mathrm{M}$ Crouzeix and $\mathrm{P}$ Raviart, Conforming and Nonconforming Finite Element Methods for Solving the Stationary Stokes Equations I, R A I R O , Vol R-3, 1973, pp $33-76$

11 M Fortin, Analysis of the Convergence of Mixed Finite Element Methods, R A I R O, Vol 11, 1977, pp 341-354

$12 \mathrm{R}$ Glowinski, Approximations exter nes, par elements finis de Lagrange d'ordre un et deux, du probleme de Dirchlet pour l'operateur biharmonique Methodes iteratives de resolution des problemes approches In Topics in Numerical Analysis, J J H MiLleR, Ed , Academic Press, New York, 1973, pp 123-171

13 P Grisvard, Singular Solutions of Elliptic Boundary Value Problems, Lecture Notes, University of Maryland (to appear)

14 L Herrmann, Finite Element Bending Analysis for Plates, J Eng Mech Div A S C E EM5, Vol 93, 1967, pp 49-83

15 L Herrmann, A Bending Analysis for Plates, Proc Conf On Matrix Methods in Structural Mechanics, AFFDL-TR-66-68, pp 577-604

$16 \mathrm{C}$ Johnson, On the Convergence of a Mixed Fintte Element Method for Plate Bending Problems, Numer Math, Vol 21, 1973, pp 43-62

17 R Kellogg and J Osborn, A regularity Result for the Stokes Problem, J Funct Anal, Vol 21, 1976, pp 397-431

18 B MERCIER, Numencal Solution of the Biharmonic Problem by Mixed Finite Elements of Class $\mathrm{C}^{0}$, Bull Un Mat Ital, Vol 10, 1974, pp 133-149

19 T Miyoshi, A Finite Element Method for the Solution of Fourth Order Partial Differential Equations, Kunamoto J Sc (Math), Vol 9, 1973, pp 87-116

$20 \mathrm{~J}$ ODEn, Some Contributions to the Mathematical Theory of Mixed Finite Element Approximations In Theory and Practice in Finte Element Structural Analysis, University of Tokyo Press, 1973, pp 3-23

21 R Rannacher, On Nonconforming and Mixed Finite Element Methods for Plate Bending Problems The Linear Case, R A I R O, Vol 13, 1979, pp 369-387

22 P Raviart and J Thomas, A Mixed Finite Element Method for 2nd Order Elliptic Problems, Lecture Notes in math , No 606, Berhn-Heidelberg-New York, Sprınger, 1977, pp 292-315

$23 \mathrm{R}$ Scholz, Approximation von Sattelpunkten mit finiten Elementen, Tagungsband, Bonn Math Schr, Vol 89, 1976, pp 53-66

24 R Scholz, A Mixed Method for 4th Order Problems using Linear Finite Elements, R A I R O, Vol 12, 1978, pp 85-90

$25 \mathrm{~J}$ Thomas, Sur l'Analyse numerique des methodes d'elements finis hybrides et mixtes, Thesis, P1erre-et-Marie-Curie, Parıs, 1977 